NEURODEGENERATIVE DISEASE

\section{A stimulating approach}

Previous studies have shown that modulating neural activity can reduce pathology associated with Alzheimer disease (AD) in mouse models of this disorder, but whether this approach can improve the AD-like cognitive deficits observed in such mice is not known. A new study reports that auditory toneinduced gamma oscillations in the auditory cortex and hippocampus reduced amyloid- $\beta(A \beta)$ pathology and improved cognitive deficits in $\mathrm{AD}$ mice. Moreover, pairing an auditory tone and a visual stimulus led to more widespread gamma entrainment and effects on such brain pathology in these animals.

A previous study from the groups involved in the new research showed that exposure to a light flickering at $40 \mathrm{~Hz}$ entrained gamma oscillations in the visual cortex of $\mathrm{AD}$ mice, reducing local levels of $A \beta$, the primary component of amyloid plaques in $\mathrm{AD}$, and altering the morphology of microglia, such that they seemed to be in a more phagocytic state, potentially indicating more $A \beta$ uptake. Here, the authors examined whether gamma entrainment by an auditory stimulus might affect other brain areas implicated in AD.

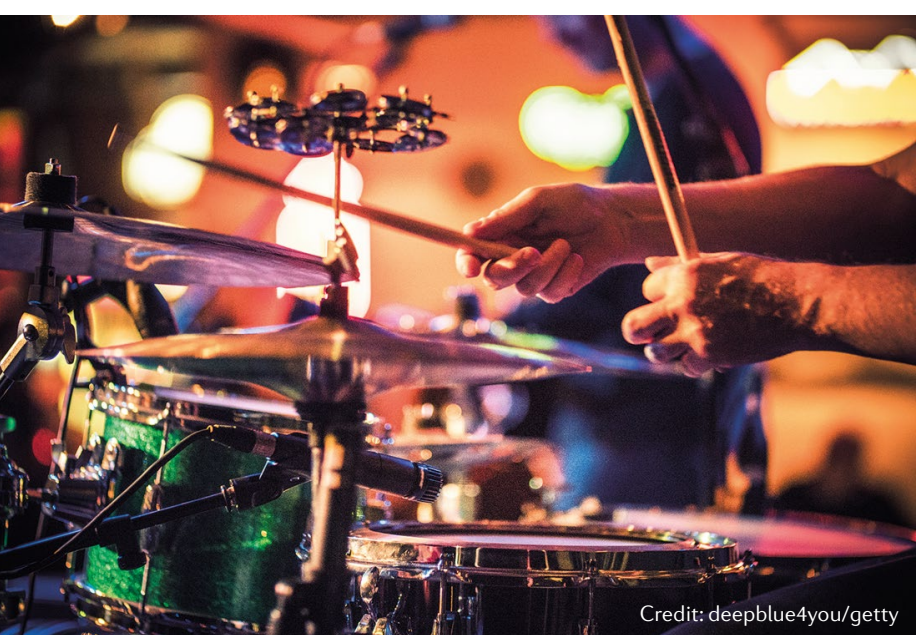

The authors first showed that wild-type mice exposed to trains of auditory tones delivered at $40 \mathrm{~Hz}$ altered single-unit firing rates in the auditory cortex, hippocampus and medial prefrontal cortex (mPFC) in such a way that they became entrained to the gamma frequency.

As such stimulation modulated hippocampal neural activity, the authors examined whether it could also affect hippocampus-dependent learning and memory, which is progressively lost in $\mathrm{AD}$. To do so, they exposed 6-month old 5XFAD mice - which start to show cognitive deficits at this time point - to bouts of auditory tone stimulation over a period of 7 days and then assessed these AD mice in novel object recognition or location tasks. Compared with no stimulation or randomly delivered auditory stimulation, the stimulation delivered at $40 \mathrm{~Hz}$ increased recognition performance in both tasks. This protocol also improved spatial memory in these mice, as assessed in the Morris water maze.

In terms of effects on brain pathology, 7 days of the auditory tone stimulation markedly reduced the soluble levels of $A \beta$ peptides in the hippocampus and the auditory cortex in 5XFAD mice, with similar effects observed in a different mouse model of AD. In addition, such stimulation reduced plaque load in these brain regions in both of the mouse models.

Auditory tone stimulation also affected microglia. After stimulation, 5 XFAD mice showed a $\sim 60 \%$ increase in microglial number in the auditory cortex and hippocampal CA1 region, morphological changes in these cells that were consistent with a more phagocytic state and an increase in microglia-A $\beta$ co-staining. Astrocytes may also play a part in clearing $A \beta$ via the vasculature, and the brains of stimulated mice showed increases in astrocyte number, blood vessel diameter and the level of lipoprotein receptor-related protein 1 (a protein involved in transporting amyloid through the brain endothelium). Together, these data suggest that $40 \mathrm{~Hz}$ auditory stimulation is associated with an upregulation of $A \beta$ clearance pathways in the brain.

In the last phase of the study, the authors examined the effects of combining auditory tone stimulation with their previously reported visual stimulation approach, first showing that the combined approach could entrain neural activity to gamma oscillations in the auditory cortex, hippocampus and $\mathrm{mPFC}$.

Strikingly, the combined approach, unlike either approach alone, could elicit morphological changes in microglia not just in the auditory cortex and hippocampus but also in the mPFC. Moreover, these changes included clustering of microglia around plaques, an effect not observed with the single sensory-modality stimulation approaches.

The combined approach, but not the auditory or visual approach alone, also reduced $A \beta$ plaque area in the $\mathrm{mPFC}$ and the levels of soluble and insoluble forms of $A \beta$. Last, using a whole-brain approach, the authors found that 5XFAD mice showed decreases in plaque number and area across the neocortex following exposure to the combined stimulus.

Together, these data reveal that auditory tone stimulation can affect not only $\mathrm{A} \beta$ pathology but also cognitive function in mouse models of $\mathrm{AD}$, and that a multi-sensory stimulation approach has even more profound effects on $\mathrm{AD}$-associated pathology in mice.

Darran Yates

ORIGINAL ARTICLE Martorell, A. J. et al. Multi-sensory gamma stimulation ameliorates Alzheimer's-associated pathology and improves cognition. Cell. https://doi.org/10.1016/j.cell. 2019.02.014 (2019) 